\title{
Applicability of 'glume color' controlled by $R$ g-B1 locus as a field-marker to detect $G l u-B 3$ alleles encoding low-molecular-weight glutenin subunits in common wheat
}

\author{
Kiyoshi Fujii*1,4), Takako Tsuji(1), Tomofumi Yoshida' ${ }^{1)}$, Wakako Maruyama-Funatsuki²) \\ and Tatsuya M. Ikeda ${ }^{3)}$ \\ 1) Aichi Prefectural Agricultural Research Center, 1 Sagamine, Yazako, Nagakute, Aichi 480-1193, Japan \\ 2) National Agricultural Research Center for Hokkaido Region, National Agriculture and Food Organization, 1 Hitsujigaoka, Toyohira, \\ Sapporo, Hokkaido 062-8555, Japan \\ 3) National Agricultural Research Center for Western Region, National Agriculture and Food Organization, 6-12-1 Nishifukazu, \\ Fukuyama, Hiroshima 721-8514, Japan \\ 4) Present address: Department of Agriculture, Aichi Prefectural College of Agriculture, 1-2 Namimatsu, Miai, Okazaki, Aichi 444- \\ 0802, Japan
}

Glu-B3 alleles on chromosome 1BS of common wheat (Triticum aestivum L.) encoding low-molecularweight glutenin subunit are associated with dough property. Among them, Glu-B3g is known to increase dough strength. To incorporate efficiently $G l u-B 3 g$ allele into breeders' lines, DNA marker-assisted selection (DNA-MAS) is considered to be a powerful tool. In the present study, we examined the applicability of 'glume color' (GC) controlled by $R g-B 1(R g 1)$ locus on $1 \mathrm{BS}$ as a 'field-marker' to detect Glu-B3 alleles. Three populations of $\mathrm{F}_{4}$ breeding lines were used. In each cross, both genotypes of $G l u-B 3$ and $R g-B 1$ loci of each parent were different. Glu-B3 allele-specific DNA markers were applied to determine the genotype of Glu-B3 locus of each line. The genotype of $R g-B 1$ locus of each line was visually determined by GC. Significantly tight cosegregations between $G l u-B 3$ and $R g-B 1$ loci were found in all the populations of breeding lines. It is considered that GC controlled by $R g-B 1$ locus is a useful 'field-marker' to detect $G l u-B 3$ alleles, as it is less time-consuming and more cost effective than DNA-MAS. By using the 'field-marker', breeders can select elite lines carrying Glu-B3g allele in breeding fields only by observing the GC of each line/plant without laboratorial procedures for DNA-MAS.

Key Words: common wheat, glutenin, dough strength, $G l u-B 3$, glume color, $R g-B 1$, field-marker.

\section{Introduction}

Seed storage proteins of common wheat (Triticum aestivum L.) present in the endosperm are composed of two major proteins, glutenins and gliadins. These are the components of wheat gluten protein, which is the most important determinant of dough properties. Glutenins are associated with dough strength and classified into high-molecular-weight (HMW) and low-molecular-weight (LMW) subunits. The HMW glutenin subunits (HMW-GSs) are encoded by the Glu-A1, Glu-B1 and Glu-D1 loci on the long arm of wheat chromosomes 1A, 1B and 1D, respectively (Payne and Lawrence 1983). While the LMW glutenin subunits (LMWGSs) are encoded by the Glu-A3, Glu-B3 and Glu-D3 loci on the short arm of these chromosomes (Jackson et al. 1983).

Recent studies have clarified that Japanese wheat cultivars with $G l u-B 3 i$ allele (e.g. 'Iwainodaichi') often do not have enough dough strength for Japanese white salted noo-

Communicated by H. Tsujimoto

Received August 2, 2010. Accepted November 18, 2010.

*Corresponding author (e-mail: Kiyoshi_fujii@pref.aichi.lg.jp) dle (Fujii et al. 2009, Ikeda et al. 2005a, 2005b, Taya et al. 2003). On the contrary, new cultivars carrying Glu-B3g allele (e.g. 'Kinunonami', 'Kitahonami' and 'Toukai 103') often show stronger dough and better sensory quality of Japanese white salted noodle compared with cultivars carrying Glu-B3i allele (Fujii et al. 2009, Ikeda et al. 2005a, 2005b, 2007, Takahashi et al. 2003, Yanagisawa et al. 2007). Extrastrong wheat varieties 'Glenlea' and 'KS831957' possess the Glu-B3g allele (Ikeda et al. 2006, Maruyama-Funatsuki et al. 2005a, 2005b, 2007). 'Glenlea' is a Canadian cultivar and ' $\mathrm{KS} 831957$ ' is a breeding line developed at Kansas University in U.S. These extra-strong dough properties of both varieties are due to the interaction effect between Glu-Dld and Glu-B3g alleles (Maruyama-Funatsuki et al. 2005a, 2005b, 2007).

In order to incorporate efficiently the $G l u-B 3 g$ allele into elite breeding lines, DNA marker-assisted selection (DNAMAS) is considered to be a quite powerful tool in wheat breeding programs. D'Ovidio et al. (1997), MaruyamaFunatsuki et al. (2005a, 2005b) and Ikeda et al. (2005a) developed DNA markers to detect Glu-B3 alleles.

Whereas, there are some difficulties to apply DNA-MAS 
Table 1. Three sets of wheat breeding lines populations used for cosegregation analysis between 'glume color' controlled by $R g$ - $B 1$ locus and Glu-B3 alleles encoding low-molecular-weight glutenin subunits and their cross parents

\begin{tabular}{|c|c|c|c|c|c|c|c|c|c|c|}
\hline \multicolumn{3}{|c|}{ Breeding line } & \multicolumn{4}{|c|}{ Seed parent } & \multicolumn{4}{|c|}{ Pollen parent } \\
\hline Cross & $\begin{array}{l}\text { Genera- } \\
\text { tion }\end{array}$ & $\begin{array}{l}\text { Number } \\
\text { of lines }\end{array}$ & Variety & $\begin{array}{l}\text { Glume } \\
\text { color }\end{array}$ & $\begin{array}{l}R g-B 1 \\
\operatorname{locus}^{a}\end{array}$ & $\begin{array}{c}\text { Glu-B3 } \\
\text { allele }\end{array}$ & Variety & $\begin{array}{l}\text { Glume } \\
\text { color }\end{array}$ & $\begin{array}{l}R g-B 1 \\
\operatorname{locus}^{a}\end{array}$ & $\begin{array}{c}\text { Glu-B3 } \\
\text { allele }\end{array}$ \\
\hline Kinuiroha/Kinunonami & $\mathrm{F}_{4}$ & 147 & Kinuiroha & red & $R g-B 1 b$ & $i$ & Kinunonami & white & $R g-B 1 a$ & $g$ \\
\hline Iwainodaichi/Kinunonami & $\mathrm{F}_{4}$ & 64 & Iwainodaichi & red & $R g-B 1 b$ & $i$ & Kinunonami & white & $R g-B 1 a$ & $g$ \\
\hline Haruibuki/Minaminokaori & $\mathrm{F}_{4}$ & 97 & Haruibuki & white & $R g-B 1 a$ & $j$ & Minaminokaori & red & $R g-B 1 b$ & $i$ \\
\hline
\end{tabular}

a Genotype of $R g-B 1$ locus was determined by 'glume color'.

in the breeding programs because DNA-MAS needs laboratorial procedures and is time-consuming as well as costly for breeders (Brennan et al. 2005, Dreher et al. 2003, Jorasch 2004, Morris et al. 2003).

$R g-B 1(R g l)$ locus controls 'glume color' of common wheat (Unrau 1950). The Glu-B3 and $R g-B 1$ loci are located on the same short arm of common wheat chromosome 1B (Jackson et al. 1983, Khlestkina et al. 2006, Worland et al. 1987). The map distance between Glu-B3 and $R g-B 1$ loci is calculated to be $17.2 \mathrm{cM}$ in common wheat in the latest wheat genetic resources database ('KOMUGI' version 3.74.2; National Bio Resources Project 2010). However, Blanco et al. (1998) reported that the map distance between the two loci was calculated to be $1.6 \mathrm{cM}$ in tetraploid durum wheat. In the present study, we examined the effectiveness and applicability of 'glume color' controlled by $R g-B 1$ locus in common wheat as a phenotypic 'field-marker' to detect Glu-B3 alleles in the breeding fields, aiming for the development of an alternative tool for breeders.

\section{Materials and Methods}

\section{Plant materials}

Three populations of common wheat breeding lines at $\mathrm{F}_{4}$ generation, which were derived from crosses between 'Kinuiroha' and 'Kinunonami', 'Iwainodaichi' and 'Kinunonami', and 'Haruibuki' and 'Minaminokaori', consisting of 147, 64 and 97 lines, respectively, were used (Table 1). In each cross, parents had different Glu-B3 allele and glume color controlled by $R g-B 1$ from those of each other (Table 1). Each population of breeding lines at $\mathrm{F}_{4}$ generation was developed in the wheat-breeding program at Aichi Prefectural Agricultural Research Center (AARC). F 1 plants of each cross were grown in the breeding fields of AARC and $F_{2}$ seeds were harvested. About $400 F_{2}$ plants of each cross were grown individually in the breeding fields of AARC. Ten seeds at $\mathrm{F}_{3}$ generation were harvested from each $\mathrm{F}_{2}$ plant, and all seeds of each cross were mixed together. About 2,000 individuals at $\mathrm{F}_{3}$ generation of each cross were grown in the breeding fields, and 147, 64 and 97 earlymaturing individuals were selected. $\mathrm{F}_{4}$ seeds were harvested from each selected plant at $\mathrm{F}_{3}$ generation. Therefore, the genotypes of Glu-B3 and $R g-B 1$ loci of each $\mathrm{F}_{4}$ line coincide with those of corresponding $\mathrm{F}_{3}$ individuals.

In order to confirm the results obtained from the linkage analysis using the three populations of breeding lines, we also examined the glume colors and genotypes of Glu-B3 and $R g-B 1$ loci of nine Japanese common wheat varieties as well as their seed and pollen parents (Table 6).

\section{Determination and genotyping of Glu-B3 locus}

The genotypes of Glu-B3 locus in parental varieties and each line were determined by the banding patterns of Glu-B3 allele-specific DNA markers as described by MaruyamaFunatsuki et al. (2005a, 2005b) and Ikeda et al. (2005a). DNA of each line was extracted from young leaves of five individuals per line, and the bulk of DNA of each line was analyzed. Sampling of leaves was performed at the tillering stage of each line in the breeding field at AARC.

\section{Determination and genotyping of $R g-B 1$ locus}

The genotype of $R g-B 1$ locus in each line was determined by glume color observations as all white for $R g-B 1 a$ homozygote, all red for $R g-B 1 b$ homozygote, and a mixture of red and white for heterozygote, observed twice in the breeding field of AARC at the ripening and maturing stages of each line. For each line, 25 individuals per line were observed.

\section{Results}

\section{Cosegregation between the two loci of Glu-B3 and Rg-B1 in} breeding lines

Among the $147 \mathrm{~F}_{4}$ breeding lines derived from a cross between 'Kinuiroha' (Glu-B3i, red-glumed and $R g-B 1 b)$ and 'Kinunonami' (Glu-B3g, white-glumed and $R g-B 1 a), 64$ lines with red glumes were all homozygous for Glu-B3i. Out of 47 lines with white glumes, 43 lines had homozygous Glu-B3g. Out of 36 lines with a mixture of red and white glumes, 32 lines were heterozygous for Glu-B3 alleles (Table 2). The chi square test showed a significantly close linkage of $G l u-B 3$ and $R g-B 1$ loci $(P<<0.001$, Table 5). The genotypes of Glu-B3 and $R g-B 1$ loci of each $\mathrm{F}_{4}$ line coincide with those of corresponding $\mathrm{F}_{3}$ individuals. The segregation ratio of $G l u-B 3$ and $R g-B 1$ loci in this population fitted the expected ratio $(3: 2: 3)$, respectively (Table 5 ).

Among $64 \mathrm{~F}_{4}$ breeding lines derived from a cross between 'Iwainodaichi' (Glu-B3i, red-glumed and $\mathrm{Rg}-\mathrm{B} 1 \mathrm{~b}$ ) and 'Kinunonami' (Glu-B3g, white-glumed and $R g-B 1 a$ ), out of 28 lines with red glumes, 26 lines had homozygotes 
Table 2. Cosegregation between $G l u-B 3$ alleles encoding LMW glutenin subunits and 'glume color' controlled by $R g$ - $B 1$ locus observed in the 147 breeding lines at $\mathrm{F}_{4}$ generation derived from the cross between 'Kinuiroha' (Glu-B3i, red-glumed, Rg-B1b) and 'Kinunonami' (Glu-B3g, white-glumed, $\operatorname{Rg}-B 1 a$ )

\begin{tabular}{|c|c|c|c|c|c|}
\hline \multirow{2}{*}{$\begin{array}{l}\text { Phenotype of glume color } \\
\text { of breeding line }\end{array}$} & \multirow{2}{*}{$\begin{array}{l}\text { Deduced genotype of } \\
R g-B 1 \text { locus }^{a}\end{array}$} & \multicolumn{3}{|c|}{ Number of lines classified by the genotype of Glu-B3 locus } & \multirow{2}{*}{ Total } \\
\hline & & Glu-B3g homo & Heterogenic & Glu-B3i homo & \\
\hline Red fixed & $R g-B 1 b$ homo & 0 & 0 & 64 & 64 \\
\hline Segregating & Heterogenic & 4 & 32 & 0 & 36 \\
\hline White fixed & $R g-B 1 a$ homo & 43 & 1 & 3 & 47 \\
\hline Total & & 47 & 33 & 67 & 147 \\
\hline
\end{tabular}

${ }^{a}$ Genotype of $R g-B 1$ locus of each line was deduced from glume color and its segregation within a line.

Table 3. Cosegregation between Glu-B3 alleles encoding LMW glutenin subunits and 'glume color' controlled by $\operatorname{Rg}$ - $B 1$ locus observed in the 64 breeding lines at $\mathrm{F}_{4}$ generation derived from the cross between 'Iwainodaichi' (Glu-B3i, red-glumed, $\left.R g-B 1 b\right)$ and 'Kinunonami' (Glu-B3g, white-glumed, $\operatorname{Rg}-B 1 a$ )

\begin{tabular}{|c|c|c|c|c|c|}
\hline \multirow{2}{*}{$\begin{array}{l}\text { Phenotype of glume color } \\
\text { of breeding line }\end{array}$} & \multirow{2}{*}{$\begin{array}{l}\text { Deduced genotype of } \\
R g-B 1 \text { locus }^{a}\end{array}$} & \multicolumn{3}{|c|}{ Number of lines classified by the genotype of Glu-B3 locus } & \multirow{2}{*}{ Total } \\
\hline & & Glu-B3g homo & Heterogenic & Glu-B3i homo & \\
\hline Red fixed & $R g-B 1 b$ homo & 0 & 2 & 26 & 28 \\
\hline Segregating & Heterogenic & 1 & 19 & 0 & 20 \\
\hline White fixed & $R g-B 1 a$ homo & 15 & 0 & 1 & 16 \\
\hline Total & & 16 & 21 & 27 & 64 \\
\hline
\end{tabular}

${ }^{a}$ Genotype of $\operatorname{Rg}-B 1$ locus of each line was deduced from glume color and its segregation within a line.

Table 4. Cosegregation between Glu-B3 alleles encoding LMW glutenin subunits and 'glume color' controlled by $R g-B 1$ locus observed in the 97 breeding lines at $\mathrm{F}_{4}$ generation derived from the cross between 'Haruibuki' (Glu-B3j, white-glumed, $R g-B 1 a$ ) and 'Minaminokaori' (Glu-B3i, red-glumed, $R g-B 1 b$ )

\begin{tabular}{|c|c|c|c|c|c|}
\hline \multirow{2}{*}{$\begin{array}{l}\text { Phenotype of glume color } \\
\text { of breeding line }\end{array}$} & \multirow{2}{*}{$\begin{array}{l}\text { Deduced genotype of } \\
R g-B 1 \text { locus }^{a}\end{array}$} & \multicolumn{3}{|c|}{ Number of lines classified by the genotype of $G l u-B 3$ alleles } & \multirow{2}{*}{ Total } \\
\hline & & Glu-B3j homo & Heterogenic & Glu-B3i homo & \\
\hline Red fixed & $R g-B 1 b$ homo & 0 & 5 & 37 & 42 \\
\hline Segregating & Heterogenic & 0 & 30 & 0 & 30 \\
\hline White fixed & $R g-B 1 a$ homo & 25 & 0 & 0 & 25 \\
\hline Total & & 25 & 35 & 37 & 97 \\
\hline
\end{tabular}

${ }^{a}$ Genotype of $R g-B 1$ locus of each line was deduced from glume color and its segregation within a line.

Table 5. Linkage analysis between $G l u-B 3$ and $R g-B 1$ loci in the three populations of breeding lines at $\mathrm{F}_{4}$ generation

\begin{tabular}{lccccc}
\hline \hline Factor & Degree of freedom & Expected ratio $^{a}$ & & \multicolumn{2}{c}{ Chi square } \\
\cline { 4 - 6 } & & & Population A $^{b}$ & Population B $^{c}$ & Population C $^{d}$ \\
\hline Glu-B3 locus & 2 & $3: 2: 3$ & 4.138 & 4.604 & $8.333^{*}$ \\
$R g-B 1$ locus & 2 & $3: 2: 3$ & 2.642 & 4.333 & 5.790 \\
Linkage & 4 & & $246.934^{* * *}$ & $118.369^{* * *}$ & $186.261^{* * *}$ \\
\hline Total & 8 & & $253.714^{* * *}$ & $127.306^{* * *}$ & $200.384^{* * *}$ \\
\hline
\end{tabular}

${ }^{a}$ Genotypes of Glu-B3 and $R g-B 1$ loci of each $\mathrm{F}_{4}$ line coinside with those of corresponding $\mathrm{F}_{3}$ individual, respectively.

${ }^{b} 147$ lines derived from the cross between 'Kinuiroha' and 'Kinunonami'.

${ }^{c} 64$ lines derived from the cross between 'Iwainodaichi' and 'Kinunonami'.

$d 97$ lines derived from the cross between 'Haruibuki' and 'Minaminokaori'.

$*$ and $* * *$ show significance at the $5 \%$ and $0.1 \%$ level, respectively.

for $G l u-B 3 i$. Out of 16 lines with white glumes, 15 lines had homozygous $G l u-B 3 g$. Out of 20 lines with a combination of red and white glumes, 19 lines were heterozygous for Glu$B 3$ alleles (Table 3). The chi square test also showed significantly close linkage between the two loci $(P<<0.001$, Table 5). The segregation ratio of $G l u-B 3$ and $R g-B 1$ loci in this population fitted the expected ratio $(3: 2: 3)$, respectively (Table 5).

Among $97 \mathrm{~F}_{4}$ breeding lines derived from a cross between 'Haruibuki' (Glu-B3j, white-glumed and $R g-B 1 a$ ) and 'Minaminokaori' (Glu-B3i, red-glumed and $R g-B 1 b$ ), out of 42 lines with red glumes, 37 lines had homozygous Glu-B3i. 
Table 6. 'Glume color' and genotypes of Glu-B3 locus of nine Japanese common wheat varieties and their parents

\begin{tabular}{|c|c|c|c|c|c|c|c|c|c|}
\hline \multirow[b]{2}{*}{ Type } & \multicolumn{3}{|c|}{ Variety } & \multicolumn{3}{|c|}{ Seed parent } & \multicolumn{3}{|c|}{ Pollen parent } \\
\hline & Name & $\begin{array}{l}\text { Glume } \\
\text { color }\end{array}$ & $\begin{array}{c}\text { Glu-B3 } \\
\text { allele }\end{array}$ & Name & $\begin{array}{l}\text { Glume } \\
\text { color }\end{array}$ & $\begin{array}{c}\text { Glu-B3 } \\
\text { allele }\end{array}$ & Name & $\begin{array}{l}\text { Glume } \\
\text { color }\end{array}$ & $\begin{array}{c}\text { Glu-B3 } \\
\text { allele }\end{array}$ \\
\hline \multirow[t]{3}{*}{1} & Kinunonami & white & $g$ & Kanto 107 & white & $g$ & Bandouwase & red & $i$ \\
\hline & Tsurupikari & white & $g$ & Bandouwase & red & $i$ & Kanto 107 & white & $g$ \\
\hline & Ayahikari & white & $g$ & Kanto 107 & white & $g$ & Kinuiroha & red & $i$ \\
\hline 2 & Hanamanten & red & $g$ & KS831957 & red & $g$ & Saikai 179 & white & $d$ \\
\hline 3 & Kinuazuma & white & $g$ & Kanto 107 & white & $g$ & Kanto 105 & red & $g$ \\
\hline \multirow[t]{2}{*}{4} & Toukai $103^{a}$ & white & $g$ & Kinunonami & white & $g$ & Saikai 184 & white & $i$ \\
\hline & Nebarigoshi & white & $g$ & Kanto 107 & white & $g$ & Chikugoizumi & white & $i$ \\
\hline \multirow[t]{2}{*}{5} & Chikugoizumi & white & $i$ & Kanto 107 & white & $g$ & Asakazekomugi & white & $i$ \\
\hline & Nishihonami & white & $i$ & Kanto 107 & white & $g$ & Minaminokomugi & white & $i$ \\
\hline
\end{tabular}

a'Tokai 103 ' was developed by DNA-MAS using Glu-B3 allele-specific DNA markers.

All of the 25 lines with white glumes had homozygotes for $G l u-B 3 j .30$ lines with a mixture of red and white glumes were all heterozygous for Glu-B3 alleles (Table 4). The chi square test also showed significantly close linkage relationship between the two loci $(P<<0.001$, Table 5). This population showed distorted segregation at Glu-B3 locus $(P<0.05$, Table 5).

The results on all the three populations of breeding lines show that there is a significantly tight cosegregation between the two loci of Glu-B3 and Rg-BI (glume color).

Cosegregation between Glu-B3 and Rg-B1 loci in varieties with different genetic backgrounds

To confirm the cosegregation between the 'glume color' and $G l u-B 3$ alleles observed in the breeding lines, we investigated the glume colors and $G l u-B 3$ alleles of nine varieties as well as their parents shown in Table 6. All of the nine varieties have at least one of the parents carrying Glu-B3g allele.

Among the nine varieties, seven varieties in Type 1, Type 2, Type 3 and Type 4 carried Glu-B3g allele. In four varieties classified as Type 1 and Type 2, the genotypes of Glu-B3 and $R g-B 1$ (glume color) loci of the seed parents were both different from those of the pollen parents. The glume colors of the Type 1 and Type 2 varieties carrying Glu-B3g allele were all the same as those of their parents harboring GluB3g allele (Table 6).

As a result, cosegregation between $G l u-B 3$ and $R g-B 1$ loci was also found in the varieties with different genetic backgrounds. This result was consistent with that observed in the three populations of breeding lines.

\section{Discussion}

All of the results obtained suggest that there is a significantly tight linkage between the two loci of Glu-B3 and $R g-B 1$ in hexaploid common wheat, although the map distance between the two loci was not determined. The results also suggest that the 'glume color' controlled by $R g-B 1$ locus is a useful phenotypic 'field-marker' to detect $G l u-B 3 g$ allele. Therefore, we actually have used the 'glume color marker' in wheat-breeding program at AARC. The usage accelerated the selection of elite breeding lines with good noodlemaking qualities.

Since the 'glume color marker' is more convenient (less time-consuming and more cost effective) than DNA-MAS, it is considered that wheat-breeders can easily select elite lines carrying desirable Glu-B3 alleles to control dough strength by observing the 'glume color' of each line or individual in the breeding fields, without any laboratorial procedures, such as DNA extraction, PCR and electrophoresis.

Glu-B3j derived from the short arm of rye (Secale cereale L.) chromosome $1 \mathrm{R}$ with the exchange of the short arm of wheat chromosome 1B. This chromosome translocation caused the loss of the Glu-B3-encoded LMW glutenin and incorporation of the Sec-1-encoded gliadins (Lelley et al. 2004). This change resulted in weak dough strength and intense dough stickiness of common wheat cultivars with the short arm of 1R (Lelley et al. 2004, Martin and Stewart 1990). Breeders can remove undesirable wheat lines with $G l u-B 3 j$ allele in the breeding fields by using the 'glume color marker'.

However, there is a limitation on the usage of the 'fieldmarker'. The 'field-marker' of Glu-B3 alleles can be used only under the conditions that the 'glume color' is controlled by $R g-B 1$ locus, as well as the genotypes of $R g-B 1$ and $G l u$ $B 3$ loci of the seed parent are both different from those of the pollen parent.

If the both parents of progenies have the same glume color and different Glu-B3 alleles (e.g. Type 4 and Type 5 in Table 6), DNA-MAS is considered to be the best tool to select lines carrying desirable Glu-B3 alleles (Ikeda et al. 2005a, Maruyama-Funatsuki et al. 2005a, 2005b). The wheat-breeding program at AARC demonstrated the effectiveness of DNA-MAS to detect $G l u-B 3$ alleles, and a cultivar 'Toukai 103' carrying Glu-B3g allele was successfully developed by DNA-MAS (see Type 4 in Table 6, Fujii et al. 2009). This cultivar shows larger micro-SDS sedimentation 
volume, stronger dough property and higher sensory quality of Japanese white salted noodle compared with those of a cultivar 'Iwainodaichi' carrying Glu-B3i allele (Fujii et al. 2009, Takata et al. 1999).

In Japan, an elite line 'Kanto 107' with Glu-B3g allele and white glume $(R g-B 1 a)$ has been used as a useful cross parent for breeding varieties with good sensory quality of Japanese white salted noodle. Therefore, most cultivars with Glu-B3g allele are white-glumed (e.g. Tsurupikari, Ayahikari and Kinunonami in Table 6) because the $G l u-B 3 g$ is commonly introduced from 'Kanto 107' (Takahashi et al. 1998, 2003, Yoshida et al. 2001).

There is an extra-ordinal case that a hard wheat cultivar 'Hanamanten' carrying Glu-B3g is red-glumed (Nakamura et al. 2007). The red glume of this cultivar is also controlled by $R g-B 1$ locus (Tsuji, personal communication). Glu-B3g and $R g-B 1 b$ alleles of 'Hanamanten' are both derived from its seed parent 'KS831957' which carries Glu-B3g and $R g$ $B 1 b$ (see Type 2 in Table 6). Beside this, a breeding line 'Kanto 105 ' is red-glumed and carries Glu-B3g (see Type 3 in Table 6). Since its pollen parent 'Shiroganekomugi' carries $G l u-B 3 d$ allele and has white glume, Glu-B3g and red glume of 'Kanto 105' may be introduced from its redglumed seed parent 'Kanto 85'.

In the present study, glume color of all the materials used is controlled by $R g-B 1$ locus. $R g-A 1(R g 3)$ and $R g-D 1(R g 2)$ loci are also known to control glume color of common wheat (Khlestkina et al. 2006). Rg-Al locus locates on the short arm of chromosome $1 \mathrm{~A}$ and $R g-D 1$ locus locates on chromosome 1D (Worland et al. 1987). $R g$ - $A 1$ locus probably links to Glu-A3 alleles encoding LMW-GSs. Further studies on the geographical distribution of genes controlling glume color of common wheat are needed to clarify the linkage relationships between glume colors and glutenin proteins (e.g. Efremova et al. 1998, Khlestkina et al. 2006).

Moreover, further analysis is necessary to elucidate precisely the map distance between $G l u-B 3$ and $R g-B 1$ loci in common wheat.

\section{Acknowledgement}

A domestic patent in Japan P4452790 [A set of primers amplifying DNA marker to detect a low-molecular-weight glutenin gene of wheat and the use of the primers] (21/4/2010, NARO and HOKUREN) was used in this study. This study was conducted by the wheat-breeding program in AARC with a special assignment and a grant by the Ministry of Agriculture, Forestry and Fisheries of Japan. A part of this study was funded by the research project for utilizing advanced technologies in agriculture, forestry and fisheries. We are grateful to the national and prefectural wheatbreeding programs in Japan for providing us some seeds of wheat varieties.

\section{Literature Cited}

Blanco,A., M.P.Bellomo, A.Cenci, C.De Giovanni, R.D’Ovidio, E. Iacono, B.Laddomada, M.A.Pagnotta, E.Porceddu, A.Sciancalepore et al. (1998) A genetic linkage map of durum wheat. Theor. Appl. Genet. 97: 721-728.

Brennan,J.P., A.Rehman, H.Raman, A.W.Milgate, D.Pleming and P.J.Martin (2005) An economic assessment of the value of molecular markers in plant breeding programs. In: $4^{\text {th }}$ Annual Conf. of the Australian Agricultural and Resource Economics Society, Coffs Harbour, Australia, 9-11 Feb.

D’Ovidio,R., M.Simeone, S.Masci and E.Porceddu (1997) Molecular characterization of a LMW-GS gene located on chromosome 1B and the development of primers specific for the Glu-B3 complex locus in durum wheat. Theor. Appl. Genet. 95: 1119-1126.

Dreher,K., M.Khairallah, J.M.Ribaut and M.Morris (2003) Money matteres (I): cost of field and laboratory procedures associated with conventional and marker-assisted maize breeding at CIMMYT. Mol. Breed. 11: 221-234.

Efremova, T.T., O.I. Maystrenko, V.S. Arbuzoba and L.I.Laikova (1998) Genetic analysis of glume colour in common wheat cultivars from the former USSR. Euphytica 102: 211-218.

Fujii,K., T.Tsuji, T.Yoshida, W.Maruyama-Funatsuki and T.M.Ikeda (2009) A new wheat cultivar 'Toukai 103' for Japanese white salted noodle with better texture, color and dough property. Res. Bull. Aichi Agric. Res. Ctr. 41:35-46.

Ikeda,T.M., E.Araki, Y.Fujita and H.Yano (2005a) Characterization of wheat seed storage protein genes for improvement in wheat flour quality. VII. Classification of low-molecular-weight glutenin subunit alleles and the development of the allele-specific DNA markers. Breed. Res. 7 (Suppl. 1\&2): 255.

Ikeda,T.M., E.Araki, Y.Fujita and H.Yano (2005b) Characterization of low-molecular-weight glutenin subunits encoded by Glu-B3 alleles in common wheat. In: Proceedings of 2005 AACC International Annual Meeting, p. 83.

Ikeda,T.M., E.Araki, Y.Fujita and H.Yano (2006) Characterization of low-molecular-weight glutenin subunit genes and their protein products in common wheat. Theor. Appl. Genet. 112: 327-334.

Ikeda,T.M., M.Yanaka and K.Takata (2007) Allelic variation of lowmolecular weight glutenin subunits and its functional importance. In: Lookhart,G.L. and N.G.PKW (eds.) Gluten Proteins 2006, AACC International Press, St. Paul, pp. 9-12.

Jackson,E.A., L.M.Holt and P.I.Payne (1983) Characterization of high molecular weight gliadin and low-molecular-weight glutenin subunits of wheat endosperm by two-dimensional electrophoresis and the chromosomal localization of their controlling genes. Theor. Appl. Genet. 66: 29-37.

Jorasch,P. (2004) Intellectual property rights in the field of molecular marker analysis. In: Lorz,H. and G. Wenzel (eds.) Biotechnology in Agriculture and Forestry, Molecular Marker System, Springer, Berlin, vol. 55.

Khlestkina,E.K., T.A.Pshenichnikova, M.S.Roder, E.A.Salina, V.S. Arbuzova and A.Borner (2006) Comparative mapping of genes for glume colouration and pubescence in hexaploid wheat (Triticum aestivum L.). Theor. Appl. Genet. 113: 801-807.

Lelley,T., C.Eder and H.Grausgruber (2004) Influence of 1BL.1RS wheat-rye chromosome translocation on genotype by environment interaction. Journal of Cereal Science 39: 313-320.

Martin,D.J. and B.G.Stewart (1990) Dough stickiness in rye-derived wheat cultivars. Euphytica 51: 11-86.

Maruyama-Funatsuki, W., K.Takata, H.Funatsuki, T.Tabiki, M.Ito, 
Z.Nishio, A.Kato, K.Saito, E.Yahata, H.Saruyama et al. (2005a) Identification and characterization of a novel LMW-s glutenin gene of a Canadian Western Extra-Strong wheat. J. Cereal Sci. 41: $47-57$.

Maruyama-Funatsuki, W., K.Takata, H.Funatsuki, T.Tabiki, M.Ito, Z.Nishio, A.Kato, K.Saito, E. Yahata, H.Saruyama et al. (2005b) An LMW-s glutenin gene of a Hard Red Winter wheat is similar to an LMW-s gene of a Canadian Western Extra-strong wheat. Breed. Sci. 55: 241-246.

Maruyama-Funatsuki,W., K.Takata, T.Tabiki, M.Ito, Z.Nishio, H. Funatsuki and H.Yamauchi (2007) A specific combination of HMW and LMW glutenin subunits results in extra-strong dough properties. In: Lookhart,G.L. and N.G.PKW (eds.) Gluten Proteins 2006, AACC International Press, St. Paul, pp. 1-5.

Morris, M., K.Dreher, J.M.Ribaut and M.Khairallar (2003) Money matters (II): cost of maize inbred line conversion scheme at CIMMYT using conventional and marker-assisted selection. Mol. Breed. 11: 235-247.

Nakamura,K., Y.Uehara, T.Hosono and T.Ushiyama (2007) Breeding of a new hard wheat cultivar 'Hanamanten' for Chinese yellowalkaline noodle. J. Hokuriku society of Crop Sci. 42: 81-84.

National Agriculture and Food Research Organization (NARO) and HOKUREN (2010) Patent P4452790 (domestic in Japan) [A set of primers amplifying DNA marker to detect a low-molecular-weight glutenin gene of wheat and the use of the primers]. MaruyamaFunatsuki, W., K.Takata, H.Yamauchi, T.Tabiki, Z.Nishio, H. Saruyama and E. Yahata (inventers).

National Bio Resource Project (2010) Wheat genetic resources database 'KOMUGI' version 3.74.2. http://www.shigen.nig.ac.jp/ wheat/komugi/maps/markerMaps.jsp
Payne,P.I. and G.J.Lawrence (1983) Catalogue of alleles for complex gene loci, Glu-A1, Glu-B1 and Glu-D1 which code for high molecular weight subunits of glutenin in hexaploid wheat. Cereal Res. Commun. 11: 29-35.

Takahashi,T., S.Orimo, A.Narizuka, Y.Saito, M.Osawa and Y.Obuchi (1998) A new wheat variety, Gunma W2. Res. Bull. Gunma Agric. Exp. Sta. 4: 19-27.

Takahashi,T., S.Orimo, A.Narizuka, Y.Saito, M.Osawa and Y.Obuchi (2003) Breeding of a new wheat variety 'Kinunonami'. Res. Bull. Gunma. Agric. Exp. Sta. 8: 1-7.

Takata,K., H.Yamauchi, N.Iriki and T.Kuwabara (1999) Prediction of bread-making quality by prolonged swelling SDS-sedimentation test. Breed. Sci. 49: 221-223.

Taya, S., T. Tohnooka, M. Seki, M.Taira, T.Tsutsumi, K. Ujihara, A.Sasaki, R.Yoshikawa, M.Fujita, Y.Taniguchi et al. (2003) New wheat cultivar "Iwainodaichi". Bull. Natl. Agric. Res. Cent. Kyushu Okinawa Reg. 42: 1-18.

Unrau,J. (1950) The use of monosomes and nullisomes in cytogenetic studies in common wheat. Sci. Agric. 30: 66-89.

Worland,A.J., M.D.Gale and C.N.Law (1987) Wheat genetics-Chromosome maps and gene location-. In: Lupton,F.G.H. (ed.) Wheat Breeding, Chapman and Hall, London \& New York, pp. 158-165.

Yanagisawa,A., Y.Yoshimura, Y.Amano, S.Kobayashi, T.Nishimura, K. Nakamichi, K.Araki, K. Tanifuji, T.Tabiki, K. Mikami et al. (2007) A new winter wheat variety 'Kitahonami'. Bull. Hokkaido. Prefect. Agric. Exp. Stn. 91: 1-13.

Yoshida,H., C.Kiribuchi-Otobe and T.Yanagisawa (2001) Breeding of a new wheat cultivar 'Ayahikari'. Bull. Natl. Agric. Res. Cent. 34: $17-35$. 